\title{
Terapia por Contensão Induzida e Treino Mental na Funçáo de Membro Superior Pós-AVC
}

\author{
Constraint-Induced Movement Therapy and Mental Practice to Improve Arm Function In Stroke Patients \\ Aline Oliveira Siqueira ${ }^{1}$, Richelma de Fátima de Miranda Barbosa ${ }^{2}$
}

\begin{abstract}
RESUMO
Introduçáo. Acidente Vascular Cerebral (AVC) é a segunda principal causa de incapacidade e morte no mundo. O sinal clínico mais comum é a hemiparesia, que compromete a execução das atividades da vida diária (AVD’S). Objetivo. Verificar o uso da terapia de contensão induzida e do treino mental na funcionalidade de membro superior pós-AVC. Método. Vinte pacientes hemiparéticos, pós-AVC, 45-74 anos, randomizados aleatoriamente em três grupos: Grupo I(Treino mental) com 6 pacientes, Grupo II(Terapia por Contensão Induzida- TCI) com 6 pacientes e Grupo controle (Cinesioterapia) com 8 pacientes. Antes e após o tratamento dos grupos, foram feitas avaliaçôes com a Escala de Fulg-Meyer, goniometria e Medida de Independência Funcional (MIF). Tratou-se por 4 meses, 2 vezes na semana, 30 minutos cada grupo. Resultado. Após as intervençóes observou-se com o Fulg-Meyer, que mede o comprometimento motor do membro superior (MMSS) melhora na mobilidade e função motora de MMSS (intragrupos) em todos os grupos, porém o grupo treino mental obteve melhor pontuação (intergrupo); na goniometria o grupo cinesioterapia obteve melhor resultado em punho/dedos (intragrupo). Na MIF, que avalia a funcionalidade nas AVD'S, o grupo Treino Mental obteve melhor resultado (intragrupo/intergrupo). Conclusáo. $\mathrm{O}$ grupo treino mental demonstrou maiores resultados na funcionalidade de membro superior após AVC.
\end{abstract}

Unitermos. Acidente Vascular Cerebral, Reabilitação, Hemiparesia, Plasticidade Neuronal.

Citação. Siqueira AO, Barbosa RFM. Terapia por Contensão Induzida e Treino Mental na Função de Membro Superior Pós-AVC.

Trabalho realizado na Universidade do Estado do Pará (UEPA), Santarém-PA, Brasil.

1.Fisioterapeuta, Santarém-PA, Brasil.

2.Fisioterapeuta, Mestre, Docente da Universidade do Estado do Pará, Santarém-PA, Brasil.

\begin{abstract}
Introduction. Stroke is the second leading cause of disability and death in the world. The most common clinical sign is hemiparesis, which undermines the performance of activities of daily living (ADL's). Objective. Analise the constraint-induced movement therapy and mental practice to improve arm function in stroke patients. Method. Twenty hemiparetic patients, post-stroke, 45-74 years, randomly randomized into three groups: Group I (mental training) with 6 patients, Group II (Therapy Constraint-induced movement-TCI) with 6 patients and control group (kinesiotherapy) with 8 patients. Before and after treatment of the groups were assessed with the Fulg Meyer Scale, goniometry and Functional Independence Measure (FIM). The intervention carried for 4 months, 2 times a week for 30 minutes. Results. In FulgMeyer, which measures motor impairment of the upper limb (UL) affected an improvement in mobility and upper limb motor function (intragroup) in all groups, but the mental training group achieved better scores (intergroup) in goniometry group kinesiotherapy best result obtained in hand/fingers (intragroup). In MIF, which evaluates the functionality ADL'S, the Mental Training group had the best results (intragroup/intergroup). Conclusion. The mental training group showed better results in rehabilitation.
\end{abstract}

Keywords. Stroke, Rehabilitation, Paresis, Neuronal Plasticity.

Citation. Siqueira AO, Barbosa RFM. Constraint-Induced Movement Therapy and Mental Practice to Improve Arm Function In Stroke Patients. 


\section{INTRODUÇÃO}

Segundo a Organizaçẫo Mundial de Saúde mais de 15 milhóes de pessoas são acometidas por Acidente Vascular Cerebral (AVC) por ano no mundo, e aproximadamente cinco milhóes de pessoas morrem e a mesma proporção permanece com sequelas físicas e funcionais permanentes ${ }^{1}$.

O Brasil ocupa o sexto lugar no ranking mundial de mortalidade e incapacidade gerada pelo $\mathrm{AVC}^{2}$. Dentre as incapacidades funcionais a hemiparesia acomete a maioria dos sobreviventes principalmente com comprometimento da extremidade superior impactando na realização de suas atividades de vida diária. Devido a este cenário de limitação física e funcional, os pacientes apresentam uma tendência de executar tarefas usando seus membros hígidos e, assim, evitam o uso do membro acometido, resultando em um fenômeno de "não-uso aprendido"3.

Diante destas incapacidades funcionais geradas após um AVC, principalmente no que tange a recuperação e aprendizagem motora de membros superiores, novas técnicas estão sendo preconizadas pela literatura científica à luz da neuroplasticidade que afirma que pacientes adultos com Acidente Vascular Cerebral demonstram alteraçôes funcionais na excitabilidade cortical, taxa metabólica, fluxo sanguíneo e reorganização do mapa cortical durante terapias de reabilitação motora ${ }^{4}$. Dentre estas, os achados científicos apontam a Terapia de Contensão Induzida (TCI) e o treino mental como promotoras de uma influência positiva na recuperação da funcionalidade do membro superior pós- AVC.

A Terapia por Contensão Induzida (TCI) é caracterizada pela restrição do membro superior não afetado através de um "splint" de posicionamento ventral, associada a um programa intensivo de treinamento funcional do membro superior hemiparético em 90\% do dia, com utilização do shaping, uma técnica comportamental 5 . Seus benefícios envolvem: melhora funcional da reorganização cortical relacionada à melhora clínica da mão, recuperação motora de indivíduos hemiparéticos crônicos, melhora da destreza, motricidade residual do membro superior hemiparético e independência funcional ${ }^{6}$.

O Treino Mental (TM), algumas vezes chamado de "imaginário motor", é uma técnica que se utiliza da imaginação do movimento antes de ser realizado com o lado hemiparético, através de imagens internas (treino cinestésico) ou externas (treino visual). $\mathrm{O}$ treino mental aumenta a aprendizagem motora e as mesmas estruturas neurais musculares são ativadas quando os movimentos são praticados mentalmente como durante a prática física das mesmas habilidades, principalmente na reabilitação funcional de membro superior pós-AVC ${ }^{7-9}$.

Apesar da grande quantidade de estudos relacionados à reabilitação em pacientes com AVC, poucos envolvem os efeitos das referidas técnicas de forma isolada, e dentre estes, o tamanho amostral é relativamente pequeno. Com isso o presente estudo se propôs a comparar a eficácia das técnicas TCI e treino mental na funcionalidade de membro superior em pacientes hemiparéticos crônicos por AVC em uma unidade de saúde de reabilitação no município de Santarém-Pará.

\section{MÉTODO}

\section{Local e Amostra}

Trata-se de um estudo experimental, exploratório, de caráter quantitativo e comparativo, do tipo série de casos. Executado após a aprovação pelo Comitê de Ética e pesquisa em seres humanos da Universidade do Estado do Pará-UEPA/Santarém-Pará, conforme protocolo $\mathrm{n}^{\circ}$ 083/2010 e mediante a assinatura do Termo de Consentimento Livre e Esclarecido pelos participantes da pesquisa.

Foram selecionados 20 pacientes pós- acidente vascular cerebral, da Unidade de Ensino e Assistência em Saúde do Baixo Amazonas (UEASBA), da Clínica Fisiotap e pacientes que tiveram alta do Hospital Municipal de Santarém, no período de Maio a Setembro de 2011, com idade entre 45 e 74 anos; de ambos os sexos; hemiparesia crônica de predomínio braquial pós- AVC; tempo de lesão de até 1 ano; espasticidade de membro superior $<2$ na Escala de Arshworth Modificada; amplitude de movimento de extensão ativa de punho de $20^{\circ}$ e de extensão ativa de dedos de $10^{\circ}$.

Sendo excluídos pacientes com lesões cerebrais múltiplas, com comprometimento visual e auditivo; espasticidade em membros superiores maior que grau 2 na Escala de Arshworth Modificada; pacientes hemiplégicos; déficits cognitivos graves; crises convulsivas recorrentes e hipertensão arterial descontrolada. 


\section{Avaliaçáo}

Todos os pacientes foram avaliados, por um avaliador cego treinado previamente pelos pesquisadores e após o recebimento de envelopes lacrados contendo os instrumentos de avaliação para cada paciente, através de uma ficha clínica-epidemiológica, pela Escala de Arshworth Modificada para pontuação do tônus muscular do membro superior parético, e pela goniometria nos seguintes pontos anatômicos de membro superior acometido (acrômio, olécrano e processo estilóide do rádio e ulna) demarcados previamente com adesivos, em decúbito dorsal, lateral direito ou esquerdo para quantificar a amplitude dos movimentos de flexão de ombro, cotovelo e punho/ dedos do hemicorpo lesionado.

Avaliou-se também o comprometimento motor do membro superior afetado pela escala de Fulg- Meyer, nos escores relacionados ao membro superior, com pontuação de 66 pontos e classificada de acordo com o nível do comprometimento motor como: até 30 pontos (severo), 30-49 pontos (moderado) e 50-66 pontos (leve) ${ }^{10}$.

A Medida de Independência Funcional (MIF) foi utilizada para avaliar a independência funcional nas atividades de vida diária com a utilização do membro superior afetado, onde avaliamos apenas os escores do item cuidados pessoais, pois estão mais relacionados com o membro superior afetado, com pontuação de 6 a 42 pontos, divididos em quatro subscores na pontuação geral: 6 pontos (dependência completa); 7 - 19 pontos (assistência de 50\%); 20 - 34 pontos (assistência de 25\%) e $35-42$ pontos (independência completa).

\section{Randomizaçáo}

Após um período de uma semana da avaliação prévia, os pacientes selecionados foram randomizados pelos avaliadores cegos que eram pesquisadores do ambiente acadêmico e não faziam parte do corpo clínico da UEASBA, de forma cega, através da entrega aleatória de envelopes opacos fechados aos pacientes, contendo os adesivos de identificação dos grupos e as descrições dos 3 grupos de intervenção: Grupo I, Grupo II, e Grupo III. Todos os pacientes foram informados sobre os reais objetivos do estudo, e estavam cientes que iriam participar de protocolos de reabilitação funcional para recuperação motora de membro superior após Acidente Vascular Cerebral.

\section{Procedimentos}

Todos os grupos de estudo realizaram as referidas terapias durante 4 meses, 2 vezes por semana, com duração de 30 minutos cada sessão, realizada na UEASBA/ STM, pelos pesquisadores, em um consultório individual, livre de ruídos, com boa iluminação e contendo uma maca, mesa, cadeira com encosto e os objetos que simulariam as atividades funcionais.

O grupo I foi composto por 6 pacientes submetidos ao protocolo de treino mental ${ }^{11}$, onde os pacientes mentalizavam por 30 minutos as seguintes atividades funcionais com o membro superior acometido de acordo com o comando verbal do terapeuta: Pegar uma colher e levá-lo à boca; Pegar um copo com e sem água e levá-lo à boca; Pegar uma escova de dente e escovar os dentes; Pegar uma escova de cabelo e pentear os cabelos; Vestir uma camisa sem e com botóes; Vestir uma calça sem e com botôes e Calçar sapato, e durante 10 minutos, realizava as tarefas funcionais citadas de forma ativa.

O grupo II foi composto por 6 pacientes, sendo aplicado o protocolo de Terapia por contensão induzi$\mathrm{da}^{5}$ que consistiu na restrição do membro superior não-afetado por um splint de posicionamento ventral para imobilização do ombro e cotovelo, durante os 5 dias da semana, 5 horas por dia, focando a realização ativa e repetitiva das tarefas funcionais supracitadas com o membro superior comprometido. Durante as sessões de fisioterapia, o paciente executava por 30 minutos as tarefas funcionais sob supervisão do terapeuta, e em 10 minutos recebia orientações sobre o diário de exercícios funcionais domiciliares.

O grupo III composto por 8 pacientes submetidos a um protocolo de cinesioterapia passiva para o membro superior por 30 min e a realizaçáo ativa das tarefas funcionais supracitadas com o membro superior hemiparético por $10 \mathrm{~min}$. Sendo que todos os grupos realizaram as atividades em sedestaçáo, à frente de uma mesa, onde estavam dispostos os objetos para as atividades de vida diária.

Os pacientes foram reavaliados pelo avaliador cego em todas as variáveis de desfecho primário (amplitude de movimento) e secundário (EFM, MIF).

\section{Análise Estatística}

Para a análise dos resultados da EFMM foram 
quantificados e comparados os escores de comprometimento motor pré e pós-aplicação dos exercícios de cada grupo. Foi verificada a média da amplitude de movimento através da goniometria do membro superior afetado pré e pós-tratamento fisioterapêutico das articulaçôes de ombro, cotovelo e punho/dedos de cada grupo. Além destes, a MIF, onde foi verificada a média do ganho funcional nas atividades de vida diária. Todos os dados foram representados pelo programa Microsoft Office Excel 2007, através de tabelas. Foram utilizados os testes t de Student e teste ANOVA (um critério) sendo considerado como valor de significância estatística o valor de $\mathrm{p}<0,05$.

\section{RESULTADOS}

Numa amostra total de 20 pacientes hemiparéticos crônicos por AVC na UEASBA/STM - Pará, o presente estudo foi predominantemente feminino em $30 \%$ dos casos, com idade prevalente na faixa etária de 55-60 anos (30\%) com média de 55 anos. Destes, 14 pacientes (70\%) apresentaram AVC do tipo isquêmico, com tempo médio de lesão de 3 a 6 meses (35\%), sendo que o comprometimento do hemicorpo direito predominou em $80 \%$ da amostra (Tabela 1). Em relação a cada grupo (Tabela 2), no grupo I houve uma média de idade de 54.50 anos e tempo de lesão médio de 4,83 meses. No grupo II, houve uma média de idade de 55.67 anos e tempo médio de lesão de 4.67 meses, enquanto que no grupo III a média de idade foi de 55.20 anos e tempo de lesão de 5,90 meses, sem relevância estatística intergrupos.

$\mathrm{Na}$ análise das diferenças das pontuaçóes intragrupos na admissão e alta pela EFMM (Tabela 3), através do teste t Student, considerando-se um p-valor $<0,05$, houve significância estatística nos seguintes escores da EFMM: mobilidade de membro superior e função motora de membro superior em todos os grupos de estudo, significando que os participantes conseguiram realizar tarefas como alcançar o alvo à frente; tocar o alvo e o levar á boca ou levar a mão acima da cabeça após os protocolos. $\mathrm{Na}$ análise intergrupos, através do teste ANOVA (um critério) (Tabela 3), considerando-se um p-valor $<0,05$, houve significância estatística na comparação do grupo I em relaçâo aos outros grupos nos seguintes escores: mobilidade de membro superior e função motora de membro superior, mostrando maior relevância em relação ao com-

Tabela 1

Características epidemiológicas da amostra de acordo com os grupos do estudo

\begin{tabular}{|c|c|c|c|c|c|c|c|c|}
\hline \multirow[t]{2}{*}{ Variáveis } & \multicolumn{2}{|c|}{ Grupo I $(\mathrm{N}=6)$} & \multicolumn{2}{|c|}{ Grupo II $(\mathrm{N}=6)$} & \multicolumn{2}{|c|}{ Grupo III $(\mathrm{N}=8)$} & \multicolumn{2}{|c|}{ Geral $(\mathrm{N}=20)$} \\
\hline & $\mathbf{n}$ & $\%$ & $\mathbf{n}$ & $\%$ & n & $\%$ & $\mathbf{n}$ & $\%$ \\
\hline \multicolumn{9}{|l|}{ Faixa-etária } \\
\hline $45-50$ & 02 & 33.33 & 01 & 16.67 & 02 & 25.00 & 05 & 25.00 \\
\hline $50-55$ & 01 & 16.67 & 02 & 33.33 & 02 & 25.00 & 05 & 25.00 \\
\hline $55-60$ & 02 & 33.33 & 02 & 33.33 & 02 & 25.00 & 06 & 30.00 \\
\hline $60-65$ & 01 & 16.67 & 01 & 16.67 & 02 & 25.00 & 04 & 20.00 \\
\hline \multicolumn{9}{|c|}{ Comprometimento (Hemicorpo) } \\
\hline Direito & 05 & 83.33 & 04 & 66.67 & 07 & 87.50 & 16 & 80.00 \\
\hline Esquerdo & 01 & 16.67 & 02 & 33.33 & 01 & 12.50 & 04 & 20.00 \\
\hline \multicolumn{9}{|l|}{ Tipo de lesáo } \\
\hline Hemorrágico & 01 & 16.67 & 01 & 16.67 & 04 & 50.00 & 06 & 30.00 \\
\hline Isquêmico & 05 & 83.33 & 05 & 83.33 & 04 & 50.00 & 14 & 70.00 \\
\hline \multicolumn{9}{|l|}{ Tempo de lesáo } \\
\hline 0 a 3 & 03 & 50.00 & 02 & 33.33 & 01 & 12.50 & 06 & 30.00 \\
\hline 3 a 6 & 01 & 16.67 & 03 & 50.00 & 03 & 37.50 & 07 & 35.00 \\
\hline 6 a 9 & 02 & 33.33 & 01 & 16.67 & 00 & 0.00 & 03 & 15.00 \\
\hline 9 a 12 & 00 & 0.00 & 00 & 0.00 & 04 & 50.00 & 04 & 20.00 \\
\hline
\end{tabular}


prometimento motor no grupo I (Treino mental) que evoluiu de um comprometimento motor moderado para leve de acordo com a EFMM. Enquanto que o grupo II (TCI) evolui de severo para moderado e o grupo III (cinesioterapia) permaneceu com comprometimento motor moderado na escala de Fulg-Meyer.

A análise dos graus da amplitude de movimento ativa (ADM) do membro superior hemiparético, para os movimentos de flexão de ombro, cotovelo, punho e dedos durante a admissão e alta nos grupos de intervenção, na análise intergrupos pelo teste ANOVA considerando-se um $\mathrm{p}$-valos $<0,05$, não houve significância estatística na comparaçáo dos grupos. A análise intragrupos pelo teste t Student evidenciou relevância estatística no ganho da ADM ativa nas articulaçóes de punho e dedos em todos os grupos de estudo, porém, houve relevância altamente significativa na ADM ativa de ombro no grupo III (cinesioterapia) (Tabela 4).

Tabela 2

Comparação dos resultados da média de idade e tempo de AVC de acordo com os grupos do estudo

\begin{tabular}{ccccc}
\hline \multirow{2}{*}{ Variáveis } & \multicolumn{3}{c}{ Grupos } & \multirow{2}{*}{ p $^{\text {ANovA }}$} \\
\cline { 2 - 4 } & I (Treino mental) & II (TCI) & III (Cinesioterapia) & 0.9485 \\
\hline $\begin{array}{c}\text { Idade (anos) } \\
\text { Tempo AVC } \\
\text { (meses) }\end{array}$ & $54.50 \pm 6.57$ & $55.67 \pm 5.92$ & $55.20 \pm 6.18$ & 0.1214 \\
\hline
\end{tabular}

$\mathrm{p}^{\mathrm{ANOVA}}=$ Resultados do teste Anova (um critério) para análise intergrupos

Tabela 3

Comparação dos resultados obtidos da Escala de Fulg-Meyer, antes e após as intervençōes, intralintergrupos do estudo

\begin{tabular}{|c|c|c|c|c|c|c|c|c|c|c|}
\hline \multirow{3}{*}{ Fulg- Meyer } & \multicolumn{9}{|c|}{ Grupos } & \multirow{3}{*}{$\mathbf{p}^{\mathrm{ANOVA}}$} \\
\hline & \multicolumn{3}{|c|}{ I (Treino mental) } & \multicolumn{3}{|c|}{ II (TCI) } & \multicolumn{3}{|c|}{ III (Cinesioterapia) } & \\
\hline & Adm & Alt & Dif & Adm & Alt & Dif & Adm & Alt & Dif & \\
\hline I- Mobilidade & $13.7 \pm 2.7$ & $15.7 \pm 0.8$ & $2.0 \pm 2.5$ & $8.0 \pm 2.2$ & $13.3 \pm 2.7$ & $5.3 \pm 1.6 *$ & $7.8 \pm 2.5$ & $13.0 \pm 2.8$ & $5.3 \pm 1.0 *$ & $0.0054^{*}$ \\
\hline I.I- Dor & $8.7 \pm 2.7$ & $11.3 \pm 3.3$ & $2.7 \pm 2.4$ & $8.0 \pm 2.8$ & $10.0 \pm 3.8$ & $2.0 \pm 1.8$ & $12.5 \pm 2.3$ & $14.1 \pm 2.6$ & $1.6 \pm 1.7$ & 0.6273 \\
\hline II-Sensibilidade & $11.8 \pm 0.4$ & $11.8 \pm 0.4$ & $0.0 \pm 0.0$ & $11.8 \pm 0.4$ & $12.0 \pm 0.0$ & $0.2 \pm 0.4$ & $12.0 \pm 0.0$ & $12.0 \pm 0.0$ & $0.0 \pm 0.0$ & 0.3288 \\
\hline $\begin{array}{c}\text { III- Funçáo } \\
\text { Motora de MMSS }\end{array}$ & $37.7 \pm 5.7$ & $52.7 \pm 3.6$ & $15.0 \pm 3.4^{*}$ & $29.3 \pm 8.5$ & $41.5 \pm 8.6$ & $12.2 \pm 4.2^{*}$ & $33.8 \pm 6.0$ & $44.1 \pm 5.2$ & $10.4 \pm 1.7^{*}$ & $0.0425^{*}$ \\
\hline $\begin{array}{l}\text { IV-Coordenaçáo/ } \\
\text { Velocidade }\end{array}$ & $4.2 \pm 0.8$ & $5.3 \pm 0.8$ & $1.2 \pm 1.0$ & $3.3 \pm 1.4$ & $4.3 \pm 1.0$ & $1.0 \pm 0.6$ & $2.4 \pm 0.5$ & $3.8 \pm 0.5$ & $1.4 \pm 0.5$ & 0.6320 \\
\hline $\begin{array}{l}\text { Pontuaçáo } \\
\text { Total }\end{array}$ & $76.0 \pm 10.1$ & $96.8 \pm 7.5$ & $20.8 \pm 4.4^{*}$ & $60.5 \pm 12.4$ & $81.2 \pm 14.8$ & $20.7 \pm 6.2^{*}$ & $68.4 \pm 6.3$ & $87.0 \pm 5.9$ & $18.6 \pm 1.8^{*}$ & 0.5683 \\
\hline
\end{tabular}

Adm=Admissão; Alt=Alta; Dif=Diferença entre os períodos de admissão e alta; $\mathrm{p}^{\mathrm{ANOVA}}=$ Resultados do teste Anova (um critério) para análise intergrupos. ${ }^{*} \mathrm{p}<0,05$

Tabela 4

Comparação dos resultados obtidos da goniometria de membro superior, antes e após as intervençōes, intralintergrupos do estudo

\begin{tabular}{|c|c|c|c|c|c|c|c|c|c|c|}
\hline \multirow{3}{*}{ Goniometria } & \multicolumn{9}{|c|}{ Grupo } & \multirow{3}{*}{$\mathbf{p}^{\mathrm{ANOVA}}$} \\
\hline & \multicolumn{3}{|c|}{ I (Treino mental) } & \multicolumn{3}{|c|}{ II (TCI) } & \multicolumn{3}{|c|}{ III (Cinesioterapia) } & \\
\hline & Adm & Alt & Dif & Adm & Alt & Dif & Adm & Alt & Dif & \\
\hline Ombro & $62.9 \pm 22.7$ & $85.3 \pm 18.6$ & $22.4 \pm 11.6$ & $46.2 \pm 15.7$ & $66.4 \pm 21.7$ & $20.2 \pm 11.4$ & $46.1 \pm 4.7$ & $65.1 \pm 3.8$ & $19.0 \pm 4.0^{*}$ & 0.9287 \\
\hline Cotovelo & $85.7 \pm 20.1$ & $109.8 \pm 21.7$ & $24.2 \pm 6.3$ & $87.4 \pm 25.6$ & $105.4 \pm 22.1$ & $18.0 \pm 5.7$ & $80.6 \pm 16.0$ & $88.6 \pm 29.0$ & $8.0 \pm 17.1$ & 0.0641 \\
\hline Punho/dedos & $45.6 \pm 9.5$ & $66.2 \pm 11.6$ & $22.9 \pm 6.1^{*}$ & $40.4 \pm 17.7$ & $61.3 \pm 12.7$ & $20.9 \pm 13.1^{*}$ & $58.7 \pm 12.7$ & $71.9 \pm 12.3$ & $13.2 \pm 2.6^{*}$ & 0.0506 \\
\hline
\end{tabular}

Adm=Admissão; Alt=Alta; Dif=Diferença entre os períodos de admissão e alta; $\mathrm{p}^{\text {ANOVA }}=$ Resultados do teste Anova (um critério) para análise intergrupos. * $\mathrm{p}<0.05$ 
Quanto aos resultados referentes à MIF, utilizou-se o teste $\mathrm{t}$ de Student, considerando-se um $\mathrm{p}$-valor $<0,05$, para análise intragrupos nas pontuaçóes nos escores da admissão e alta em cada grupo (Tabela 5), e obtivemos significância estatística para todos os grupos. Porém na pontuaçâo geral dos grupos na MIF, o Grupo I (Treino mental) evoluiu de 16 pontos (Dependência modificada com assistência de 50\%) para 34 pontos (Dependência modificada com assistência de 25\%). O grupo II (TCI) evoluiu de 15 pontos (Dependência com assistência de $50 \%$ ) para 27 pontos (Dependência com assistência de $25 \%$ ) e o grupo III (cinesioterapia) evoluiu de dependência com assistência de 50\% (16 pontos) para Dependência com assistência de 25\% (29 pontos). Concluindo que o grupo de estudo com maior diferença de pontuação foi o grupo Treino mental. $\mathrm{Na}$ análise intergrupos pelo teste ANOVA (um critério) houve significância estatística na comparação do Grupo I(treino mental) em relação aos demais grupos nos escores arrumar-se, vestuário (MI) e na pontuação geral da MIF.

\section{DISCUSSÃO}

A recuperação após o AVC é amplamente estudada quanto à melhora funcional a qual geralmente é obtida nos três primeiros meses após a lesão, enquanto o maior ganho na recuperação da motricidade tem sido observado nos primeiros seis meses pós-AVC, pois está associado ao aumento da excitabilidade motora do hemisfério acometido $^{12}$. Sustentando as características da presente amostra composta em sua maior prevalência por indivíduos hemiparéticos crônicos, com tempo de lesão de 3 a 6 meses pós-AVC em todos os grupos de estudo, característica sugere um bom prognóstico de recuperação motora e funcional para os mesmos, fundamentando os seguintes achados funcionais nos grupos de estudo ${ }^{11,13}$. A exemplo de estudos ${ }^{14}$, em sua série de casos de pacientes pós-AVC na fase aguda, em três grupos de estudo: treino mental (1 paciente), TCI (1 paciente) e treino mental e TCI (2 pacientes) em que se observou melhora significativa na recuperação motora nos grupos de treino mental e na terapia de contensão induzida isolados, além da ativação de áreas corticais bilaterais em ambos.

A escala de Fulg-Meyer tem se mostrado como parâmetro de avaliação rotineiro nos estudos que envolvem recuperação motora e funcional de membro superior pós-AVC, pois tem demonstrado validação e confiabilidade de teste nos exames de motricidade fina e grosseira de membros superiores em indivíduos pós $\mathrm{AVC}^{15,16}$. $\mathrm{Na}$ escala de Fulg-Meyer Modificada verificou-se que houve relevância estatística em todos os grupos em relação ao comprometimento motor do membro superior parético. Estudos afirmam que um aumento de cinco pontos no domínio de função motora da EFMM corresponde a uma melhora estatisticamente significativa da função motora de membro superior hemiparético ${ }^{17}$ similar às pontuações encontradas neste estudo ${ }^{18}$.

Numa série de $\operatorname{casos}^{7}$ que utilizou a prática mental em 29 pacientes hemiparéticos crônicos por AVC, dis-

Tabela 5

Comparação dos resultados obtidos da Medida de Independência Funcional, antes e após as intervençöes, intralintergrupos do estudo

\begin{tabular}{|c|c|c|c|c|c|c|c|c|c|c|}
\hline \multirow{3}{*}{ Cuidados pessoais } & \multicolumn{9}{|c|}{ Grupos } & \multirow{3}{*}{$\mathbf{p}^{\mathrm{ANOVA}}$} \\
\hline & \multicolumn{3}{|c|}{ I (Treino mental) } & \multicolumn{3}{|c|}{ II (TCI) } & \multicolumn{3}{|c|}{ III (Cinesioterapia) } & \\
\hline & Adm & Alt & Dif & Adm & Alt & Dif & Adm & Alt & Dif & \\
\hline Alimentação & $2.8 \pm 1.5$ & $5.7 \pm 0.8$ & $2.8 \pm 1.6^{*}$ & $4.0 \pm 1.7$ & $5.8 \pm 1.2$ & $1.8 \pm 1.2$ & $4.3 \pm 1.7$ & $6.1 \pm 0.8$ & $1.9 \pm 1.0^{*}$ & 0.3026 \\
\hline Arrumar-se & $2.3 \pm 0.8$ & $5.5 \pm 0.5$ & $3.2 \pm 1.2^{*}$ & $3.0 \pm 0.6$ & $4.8 \pm 0.4$ & $1.8 \pm 0.8^{*}$ & $3.3 \pm 0.9$ & $5.1 \pm 0.8$ & $1.9 \pm 0.4^{*}$ & $0.0118^{*}$ \\
\hline Banho & $3.5 \pm 1.4$ & $6.0 \pm 1.1$ & $2.5 \pm 1.9^{*}$ & $3.3 \pm 1.6$ & $4.3 \pm 1.2$ & $1.0 \pm 0.6$ & $2.9 \pm 0.6$ & $4.1 \pm 0.8$ & $1.3 \pm 0.7^{*}$ & 0.0789 \\
\hline Vestuário (MS) & $3.5 \pm 0.8$ & $6.2 \pm 0.8$ & $2.7 \pm 1.4^{*}$ & $3.3 \pm 0.5$ & $4.8 \pm 0.4$ & $1.5 \pm 0.5^{*}$ & $3.3 \pm 0.7$ & $5.3 \pm 0.7$ & $2.0 \pm 0.8^{*}$ & 0.1233 \\
\hline Vestuário (MI) & $3.0 \pm 0.9$ & $6.2 \pm 1.0$ & $3.2 \pm 1.0^{*}$ & $2.8 \pm 0.8$ & $4.5 \pm 0.5$ & $1.7 \pm 0.5^{*}$ & $3.5 \pm 0.8$ & $4.8 \pm 0.9$ & $1.3 \pm 0.7^{*}$ & $0.0009^{*}$ \\
\hline Higiene pessoal & $3.7 \pm 1.0$ & $5.7 \pm 1.0$ & $2.0 \pm 1.3^{*}$ & $3.5 \pm 1.5$ & $5.2 \pm 1.3$ & $1.7 \pm 1.2$ & $3.6 \pm 1.4$ & $5.4 \pm 0.5$ & $1.8 \pm 1.3^{*}$ & 0.8906 \\
\hline Pontuaçáo total & $18.8 \pm 3.6$ & $35.2 \pm 1.3$ & $16.3 \pm 4.1^{*}$ & $20.0 \pm 4.4$ & $29.5 \pm 4.3$ & $9.5 \pm 2.0^{*}$ & $20.8 \pm 2.7$ & $30.8 \pm 2.3$ & $10.0 \pm 3.1^{*}$ & $0.0026^{*}$ \\
\hline
\end{tabular}

Adm=Admissão; Alt=Alta; Dif=Diferença entre os períodos de admissão e alta; $\mathrm{p}^{\mathrm{ANOVA}}=$ Resultados do teste Anova (um critério) para análise intergrupos. ${ }^{*} \mathrm{p}<0.05$ 
tribuídos em três grupos de acordo com o tempo de terapia (20 minutos, 40 minutos e 60 minutos), durante três meses com frequência de 3 dias semanais, observou-se melhora estatisticamente significativa nas pontuaçóes do Fulg-Meyer em todos os grupos, similares a outros achados ${ }^{9}$, onde evidenciou maior relevância estatística na diminuição do comprometimento motor pela EFM no grupo submetido ao treino mental. A prática repetitiva da tarefa motora quando associada ao treino mental tem sua eficácia de ativação neural aumentada em virtude da ação cognitiva focada à tarefa aprendida, assim, há repercussão na plasticidade cortical e no desempenho da recuperação motora após $\mathrm{AVC}^{19}$. Refutando nossos resultados ${ }^{9}$, um estudo com 20 pacientes crônicos após AVC, utilizou o treino mental auditivo (30 minutos) associado a repetição em massa de tarefas (1 hora), três vezes por semana e não observou melhora significativa no comprometimento motor pela escala de EFM.

Um estudo ${ }^{20}$ que comparou 13 pacientes com AVC, divididos em 2 grupos: o primeiro para tratamento com TCI e o segundo gru $\neg$ po, controle, utilizou a escala de Fulg-Meyer e a res-sonância magnética como parâmetros de avaliação. Os pacientes submetidos ao tratamento com TCI exibiram melhoria signiᄀficante no desempenho motor do membro afetado e maior ativação dos hemisférios cerebrais bilateralmente após a intervenção, especialmente contralateral a lesão, durante o movimento do membro acometido, outros autores afirmam que após a aplicação da TCI em hemiparéticos, leva a melhora na pontuação da EFM associada a ativação cortical de membro superior na ressonância magnética funcional ${ }^{21,22}$. Concordando com os achados deste estudo, em que o grupo de pacientes submetidos ao TCI, obteve melhora no comprometimento motor pós-AVC, após 4 meses de tratamento com frequência semanal de 3 dias com 30 minutos de duração. Similarmente, outros estudos que se utilizaram da terapia de contensão induzida na motricidade de membro superior de pacientes hemiparéticos após AVC, através de outras escalas avaliativas como o Teste de função motora de Wolf, verificaram melhora estatisticamente significante no comprometimento motor após a terapia ${ }^{23}$.

É válido ressaltar que a cinesioterapia passiva associada ao treino funcional repetitivo gera aprendizado motor e repercute positivamente na melhora funcional de pacientes hemiparéticos por $\mathrm{AVC}^{4}$, achados estes também descritos em nossos resultados que fortalecem a efetividade de um protocolo de cinesioterapia funcional na reabilitação de membro superior pós-AVC.

Os pacientes hemiparéticos por AVC com prejuízos na capacidade motora e atividades funcionais de membro superior, apresentam redução nas amplitudes de movimento do membro superior devido à hemiparesia. Em nosso estudo houve um ganho de amplitude de movimento do membro superior parético estatisticamente significativo em todos os grupos. Confirmado pelas bases da neurofisiologia e neuroplasticidade a respeito da reorganização do mapa cortical motor, principalmente em relação à maior representação cortical de membro superior no homúnculo de Pienfield, o que favorece a neuroplasticidade e brotamento neuronal após uma lesão diante de técnicas que estimulam a aprendizagem motora pela repetição e execução da tarefa funcional, promovendo assim maiores resultados através de atividades funcionais que promovam maior ênfase na mobilidade desta articulaçãa $^{12}$. Em outro estudo ${ }^{24}$ foi aplicado o TCI em 20 pacientes hemiplégicos crônicos após AVC, durante 2 semanas, com restrição diária associado ao treino funcional repetitivo do lado comprometido, e verificou melhora na ADM do membro superior comprometido, na espasticidade e na funcionalidade de membro superior, que se manteve após 6 meses de seguimento follow-up.

Quanto à funcionalidade mensurada através da Medida de Independência Funcional-MIF, constatou-se que o grupo submetido ao Treino mental obteve significância nos itens arrumar-se, banho e vestimenta inferior, porém sua classificação geral na admissão foi de Dependência completa e na alta do atendimento como Dependência modificada, concordando com estudo ${ }^{25}$ em que dois hemiparéticos pós-AVC, que utilizaram a MIF foram classificados como dependência modificada com assistência de $25 \%$ das tarefas, concordando com os achados da presente pesquisa. Em contrapartida, outros estudos utilizaram outras medidas de desfecho para análise da funcionalidade como Box and Block Test, ARAT, e índice de Barthel e evidenciaram recuperação funcional nos pacientes após o Treino mental ${ }^{9,11,26}$.

Após a TCI obteve-se ganhos funcionais signifi- 
cantes nos itens alimentação, arrumar-se e vestimenta inferior, sendo classificado na admissão como Dependência completa e na alta do atendimento como Dependência modificada, obtendo assim diminuição do comprometimento motor de membro superior lesado em pacientes pós-AVC. Sustentando esses dados, um estudo placebo controlado com 26 pacientes idosos crônicos por AVC, foram divididos em dois grupos (TCI e fisioterapia tradicional) por 3 semanas, constatou-se que houve aumento nos escores da pontuação da MIF relativamente significante após a terapia de contensão induzida do membro superior em relação a fisioterapia convencional ${ }^{17}$. Um estudo $^{26}$ com 52 pacientes hemiparéticos pós-AVC subdivididos em dois grupos: um grupo submetido ao TCI e outro com grupo controle, submetido à fisioterapia convencional, 5 vezes por semana, durante 15 dias, através da MIF não se observou nenhum ganho funcional estatisticamente significativo após o protocolo, refutando nossos achados, provavelmente devido ao presente estudo ter sido executado por um período de 4 meses com maior ênfase a repetição, treino funcional e aprendizagem motora. Reforçando as correntes da literatura que afirmam que a aprendizagem motora associada ao treino funcional e repetição em massa da tarefa motora, favorece a neuroplasticidade cortical ${ }^{27}$.

Quanto ao grupo III (cinesioterapia) foi verificado melhoras significativas nos itens arrumar-se, vestimenta superior, vestimenta inferior e higiene pessoal, evoluindo para dependência modificada. Retratando que a fisioterapia convencional direcionada à tarefa repercute positivamente no ganho funcional do paciente hemiparético pós-AVC, pois quanto mais precoce a intervenção melhor é o prognóstico funcional ${ }^{28}$.

O treinamento mental parece ser uma técnica complementar, porém não substitui a execução motora dos movimentos, capaz de proporcionar efeitos adicionais ao treinamento motor pela maior estimulação central, além de diversificá-lo. Pode ser aplicado com segurança e dispensa instalaçôes especiais e equipamentos, sendo um recurso simples e de baixo custo $^{29}$. Fomentado pela literatura que afirma que a recuperação motora funcional de membro superior parético após AVC deve respeitar os seguintes parâmetros: início da reabilitação o mais precoce possível, focalizar estimulação sensório-motora direcio- nada a tarefa funcional de forma repetitiva; evoluçáo contínua para tarefas mais complexas; direcionar exercícios passivos nas articulaçôes de punho e dedos para conferir mobilidade e retardar a espasticidade, e treino ativo para ombro e cotovelo, e principalmente ofertar variabilidade de estímulos ambientais exteroceptivos e proprioceptivos na execução de atividades funcionai ${ }^{30}$.

\section{CONCLUSÃO}

O estudo revelou que o grupo I (treino mental) demonstrou resultados significantes quanto aos itens mobilidade e função motora na Escala de Fulg-Meyer e quanto aos itens arrumar-se, vestir-se (membro inferior) e pontuação geral ao utilizar a MIF na funcionalidade do membro superior afetado por hemiparesia após AVC em relação aos grupos TCI e cinesioterapia.

Porém, o grupo cinesioterapia obteve maiores resultados quanto à amplitude de movimento da articulação punho/dedos (intragrupo). Quanto ao TCI apenas não obteve significância quanto à amplitude de movimento (intergrupo). Sendo assim o Grupo I (Treino Mental) obteve melhores resultados (intergrupo/intragrupo) quando comparados às técnicas TCI e cinesioterapia.

Com a finalidade de melhorar a funcionalidade do membro superior hemiplégico pós-AVC sugere-se o desenvolvimento ou aprimoramento na realização destas técnicas.

\section{REFERÊNCIAS}

1.Assis GA, Correia AGD, Vaz CJN, Lopes RD. Neuror: Sistema de realidade aumentada para reabilitaçẫo física de pacientes vítimas de Acidente Vascular Encefálico [monografia]. São Paulo; 2010, p.1-133. Disponível em: www.sbis. org.br/cbis11/arquivos/867.pdf

2.Caneda MAG, Fernandes JG, Almeida AG, Mugno FE. Confiabilidade de escalas de comprometimento neurológico em pacientes com acidente vascular cerebral. Arq Neuropsiquiatr 2006;64(3-A):690-7.

http://dx.doi.org/10.1590/S0004-282X2006000400034

3.Lee VD, Wagenaar RC, Lankhorsst GJ, Vogelaar TW, Devillé WL, Bouter LM. Forced use of upper extremity in chronic stroke patients. Stroke 1999;30:2369-75.

http://dx.doi.org/10.1161/01.STR.30.11.2369

4.Gauthier LV, Taub E, Perkins C, Ortmann M, Mark VW, Uswatte G. Remodeling the Brain Plastic Structural Brain Changes Produced by Different Motor Therapies After Stroke. Stroke 2008; 39(5):1520-25.

http://dx.doi.org/10.1161/STROKEAHA.107.502229 
5.Vaz DV, Alvarenga RF, Mancini MC, Pinto TPS, Furtado SRC, Tirado MGA. Terapia de movimento induzido pela restriçẫo na hemiplegia: um estudo de caso único. Fisioterapia e Pesquisa, São Paulo 2008;15(3):298-303.

6.Sousa RCP, Terra FR, Carbonero FC, Campos D. Terapia de Restrição e Indução do Movimento em Hemiparéticos. Rev Neurocienc 2012;20(4):604-11. 7. Page SJ, Dunning K, Hermann V, Leonard A, Levine P. Longer Versus Shorter Mental Practice Sessions for Affected Upper Extremity Movement After Stroke A Randomized Controlled Trial. Clin Rehabil 2011;25(7):627-37. http://dx.doi.org/10.1177/0269215510395793

8.Ietswaart M, Johnston M, Dijkerman HC, Joice S, Scott CL, MacWalter RS, et al. Mental practice with motor imagery in stroke recovery: randomized controlled trial of efficacy. Brain 2011;134:1373-86.

http://dx.doi.org/10.1093/brain/awr077

9.Page SJ, Murray C, Hermann V, Levine P. Retention of Motor Changes in Chronic Stroke Survivors Who Were Administered Mental Practice. Arch Phys Med Rehabil 2011;92(11):1741-5.

http://dx.doi.org/10.1016/j.apmr.2011.06.009

10.Faria I. Função do membro superior em hemiparéticos crônicos: Análise através da classificação internacional de funcionalidade, incapacidade e saúde [Dissertação]; Belo Horizonte: Universidade Federal de Minas Gerais; 2008, p.1-113.

11.Page SJ, Levine P, Leonard A. Mental practice in chronic stroke: Results of a randomized, placebo-controlled trial. Stroke 2007;38:1293-7.

http://dx.doi.org/10.1161/01.STR.0000260205.67348.2b

12.Trevisan CM, Trintinaglia V. Efeito das terapias associadas de imagem motora e de movimento induzido por restrição na hemiparesia crônica: estudo de caso. Fisioterapia e Pesquisa 2010;17(3):264-9.

http://dx.doi.org/10.1590/S1809-29502010000300014

13.Wolf SL, Winstein CJ, Miller JP, Thompson PA, Taub E, Uswatte G, et al. Retention of upper limb function in stroke survivors who have received constraint-induced movement therapy: the EXCITE randomized trial. Lancet Neurol 2008;7:33-40.

http://dx.doi.org/10.1016/S1474-4422(07)70294-6

14.Butler AJ, Page SJ. Mental Practice With Motor Imagery: Evidence for Motor Recovery and Cortical Reorganization After Stroke. Arch Phys Med Rehabil 2006;87(12):1-17. http://dx.doi.org/10.1016/j.apmr.2006.08.326

15.Duncan PW, Propst M, Nelson SG. Reliability of the Fugl-Meyer assessment of sensorimotor recovery following cerebrovascular accident. Phys Ther 1983;63:1606-10.

16.Fritz SL, Light KE, Patterson TS, Behrman AL, Davis SB. Active Finger Extension Predicts Outcomes After Constraint-Induced Movement Therapy for Individuals With Hemiparesis After Stroke. Stroke 2005;36:1172-7.

http://dx.doi.org/10.1161/01.STR.0000165922.96430.d0

17.Valente SCF, Paula EB, Abranches M, Masiero D, Chamlian TR, Lacanno SN, et al. Resultados da fisioterapia hospitalar na função do membro superior comprometido após acidente vascular encefálico. Rev Neurocienc 2006;14(3):122-6.

18.Wu C, Chen C, Tsai W, Lin K, Chou S. A Randomized Controlled Trial of Modified Constraint-Induced Movement Therapy for Elderly Stroke Survivors: Changes in Motor Impairment, Daily Functioning, and Quality of Life. Arch Phys. Med Rehabil 2007;88:273-8.

http://dx.doi.org/10.1016/j.apmr.2006.11.021

19.Page SJ, Szaflarski JP, Eliassen JC, Pan H, Cramer SC. Cortical plasticity following motor skill learning during mental practice in stroke. Neurorehabil. Neural Repair 2009;23(4):382-8.

http://dx.doi.org/10.1177/1545968308326427

20.Lin KC, Chung HY, Wu CY, Liu HL, Hsieh YW, Chen IH, et al. Constraint-induced therapy versus control interven $\neg$ tion in patients with stroke: a functional magnetic resonance imaging study. Am J Phys Med Rehabil, 2010;89:177-85.

http://dx.doi.org/10.1097/PHM.0b013e3181cf1c78

21.Szaflarski JP, Page SJ, Kissela BM, Lee J, Levine P, Strakowski SM. Cortical Reorganization Following Modified Constraint-Induced Movement Therapy: A Study of 4 Patients With Chronic Stroke. Arch Phys Med Rehabil 2006;87:1052-7.

http://dx.doi.org/10.1016/j.apmr.2006.04.018

22.Corwin Boake C, Noser EA, Ro T, Baraniuk S, Gaber M, Johnson R, et al. Constraint-Induced Movement Therapy During Early Stroke Rehabilitation. Neurorehabil Neural Repair 2007;21:14-24.

http://dx.doi.org/10.1177/1545968306291858

23.Taub E, Uswatte G, King DK, Morris D, Crago JE, Chatterjee A. A Placebo-Controlled Trial of Constraint-Induced Movement Therapy for Upper Extremity After Stroke. Stroke 2006;37:1045-9.

http://dx.doi.org/10.1161/01.STR.0000206463.66461.97

24. Siebers A, Öberg AS, Skargren E. The Effect of Modified Constraint-Induced Movement Therapy on Spasticity and Motor Function of the Affected Arm in Patients with Chronic Stroke. Physiotherapy Canada, 2006;62(4):388-396. http://dx.doi.org/10.3138/physio.62.4.388

25.Siqueira AO, Barbosa RFM. O uso do treino mental na funcionalidade de membros superiores pós-acidente vascular encefálico. $2^{\circ}$ Congresso Amazônico de Saúde e Qualidade de Vida: a Humanização no contexto da saúde; 6-10 de junho de 2011; Santarém- PA: Universidade do Estado do Pará. 2011; Editora UEPA; p.167-9.

26.Dromerick AW, Lang CE, Birkenmeier RL, Wagner JM, Miller JP, Videen TO, et al. Very Early Constraint-Induced Movement during Stroke Rehabilitation (VECTORS) A single-center RCT. Neurology 2009;73:195-201.

http://dx.doi.org/10.1212/WNL.0b013e3181ab2b27

27.Liepert J. Motor Cortex Excitability in Stroke Before and After Constraintinduced Movement Therapy. Cog Behav Neurol 2006;19(1):41-7.

http://dx.doi.org/10.1097/00146965-200603000-00005

28. Tangeman PT, Banaitis DA, Williams AK. Rehabilitation of chronic stroke patients. Arch Phys Med Rehabil 1990;71:876-80.

29.Andrade TG; Asa SKP. Prática Mental para Pacientes com Sequelas Motoras Pós Acidente Vascular Cerebral. Rev Neurocienc 2011;19(3):542-50.

30. Hlustık P, Mayer M. Paretic Hand in Stroke: From Motor Cortical Plasticity Research to Rehabilitation. Cog Behav Neurol 2006;19(1):34-40.

http://dx.doi.org/10.1097/00146965-200603000-00004 